\title{
Moeda endógena e progresso tecnológico induzido num modelo macrodinâmico pós-keynesiano ${ }^{1}$
}

\author{
Luciano Dias Carvalho ${ }^{2}$ \\ José Luís Oreiro ${ }^{3}$
}

\section{Resumo}

Este artigo tem por objetivo analisar o processo de crescimento econômico e distribuição funcional da renda em uma situação na qual o progresso tecnológico e a taxa básica de juros são endógenos e a moeda, via oferta de crédito, tem um papel preponderante na determinação da dinâmica de longo prazo da economia. Neste contexto, demonstramos diversas combinações onde ocorrem equilíbrios múltiplos e flutuações amortecidas nas principais variáveis econômicas. Além disso, para baixa parcela salarial, verificamos um predomínio de um regime de acumulação impulsionado pelos lucros e, para altas parcelas, um regime de acumulação impulsionado pelos salários.

Palavras-chave: Dinâmica Não-Linear; Equilíbrios múltiplos; Endogeneidade monetária; Progresso tecnológico endógeno; Teoria pós-Keynesiana.

\section{Abstract}

Endogenous money and induced technical progress in a post Keynesian macrodynamic model

This article intend to analyze the process of the economic growth and income distribution in an environment where technological progress and the basic rate of interest are endogenous and the money, via credit offer, has a preponderant role in the determination of economic dynamics. In this context, we demonstrate several combinations where happen multiple equilibriums and soften flotation in the main relevant variables. Besides, for low salary portions, we observed the prevalence of a profit-led accumulation regime and, for high portions, a wage-led accumulation regime.

Key words: Non-linear dynamics; Multiple equilibrium; Endogenous money supply; Endogenous technological progress.

JEL E120, O110, O420.

\section{Introdução}

Apesar da primordial importância que a Teoria Keynesiana atribui à moeda como determinante do crescimento econômico, os principais modelos de

(1) Trabalho recebido em junho de 2006 e aprovado em junho de 2007.

(2) Doutorando em Economia do Programa de Pós-Graduação em Desenvolvimento Econômico da Universidade Federal do Paraná (PPGDE/UFPR), Professor Assistente do Departamento de Economia da Universidade Estadual do Oeste do Paraná (Unioeste), Francisco Beltrão, Paraná, Brasil. E-mail: $<$ lucianodc@gmail.com>.

(3) Doutor em Economia (Instituto de Economia da Universidade Federal do Rio de Janeiro - IE/UFRJ), Professor Adjunto do Departamento de Economia da UFPR e Pesquisador do CNPq (Conselho Nacional de Desenvolvimento Científico e Tecnológico), Curitiba, Paraná, Brasil. E-mail: <joreiro@ sociais.ufpr.br> / Web-

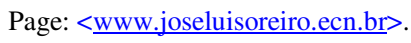


crescimento Pós-keynesianos continuam ignorando, em suas análises de longo prazo, o papel da moeda como elemento essencial do processo de acumulação de capital. ${ }^{4}$ Isso talvez se deva ao trabalho seminal de Harrod (1939), que tentou estender para o longo prazo as conclusões da Teoria Geral sem, no entanto, levar em consideração a influência da moeda sobre a dinâmica do crescimento econômico.

Assim, o desenvolvimento dos chamados modelos de primeira geração notadamente os de Domar (1946), Kalecki (1954), Kaldor (1956, 1957), Pasinetti (1962) e Robinson (1962) -, que têm em comum uma abordagem do crescimento econômico com base na distribuição funcional da renda e, implicitamente, numa plena utilização da capacidade produtiva, são indiferentes ao papel da moeda na determinação do nível de produção de longo prazo.

Essa mesma abordagem não monetária do processo de crescimento econômico pode ser encontrada nos modelos de segunda e terceira gerações, tais como os de Goodwin (1967), Rowthorn (1981), Dutt (1984, 1994), Bhaduri e Marglin (1990) e Lima (1999), que, apesar de incorporarem uma dinâmica mais complexa baseada em influências não-lineares entre as variáveis, na flexibilização do grau de utilização da capacidade produtiva e na endogenização do progresso tecnológico não levam em consideração o papel do financiamento no processo de acumulação de capital.

Uma forma aparentemente interessante de incluir a influência do sistema financeiro sobre o sistema produtivo surge com a análise dos escritos de Keynes e Schumpeter. A despeito das diferenças de visão de mundo existentes entre eles há, em ambas as teorias, certa complementaridade quanto ao papel da moeda no processo de desenvolvimento econômico. ${ }^{5}$ Diferentemente do pensamento ortodoxo que vê na moeda um mero elemento facilitador de trocas, essas duas teorias atribuem um papel importante para a moeda na dinâmica de longo prazo do sistema capitalista. Para Keynes, a distinção entre a neutralidade e a nãoneutralidade da moeda se dá através da dicotomia entre economia cooperativa e economia empresarial; já para Schumpeter, por meio dos conceitos de fluxo circular e de desenvolvimento econômico. Em ambas as abordagens, a moeda afeta no longo prazo tanto a trajetória de acumulação quanto o próprio estoque final de bens de capital.

No caso particular da visão schumpeteriana, o fluxo circular engloba todas as situações em que o equilíbrio walrasiano pode ser satisfatoriamente aplicado, enquanto o desenvolvimento econômico é a situação na qual o processo de

(4) Para o descaso dos economistas Pós-keynesianos com relação à influência da moeda no crescimento econômico ver, por exemplo, Kregel (1985) e Carvalho (1992).

(5) Para maiores detalhes, ver Vercelli (1991). 
mudança tecnológica gera perturbações no equilíbrio que não podem ser perfeitamente absorvidas pela estrutura vigente na economia.

Desse modo, no fluxo circular, a moeda não tem nenhum papel a não ser o de facilitar a circulação de mercadorias sendo, portanto, tal como apregoa a ortodoxia, um véu que encobre os mecanismos econômicos sem, no entanto, influenciá-los. Em contraste, no processo de desenvolvimento econômico, entendido como o deslocamento constante das condições de equilíbrio existentes na economia em decorrência de sucessivas inovações tecnológicas, a moeda - ou, mais especificamente, o crédito - apresenta um importante papel na viabilização do desenvolvimento através do adiantamento de recursos monetários que só serão gerados ex-post ao processo de inovação. Com efeito, em um ambiente onde predominam inovações, o papel dos bancos através do crédito bancário passa a ser de relevante importância, pois só através deles é possível introduzir no sistema econômico inovações tecnológicas.

A endogenização da oferta de moeda nos modelos Pós-keynesianos pode ser encontrada, por exemplo, em Dutt (1989, 1992), Dutt e Amadeo (1993), Lima e Meirelles (2003) e, de uma forma diferente, em Watanabe (1997). Para os primeiros, a endogeneidade da oferta de moeda se dá através da suposição de que os bancos possuem a capacidade de atender qualquer demanda adicional a uma dada taxa de juros que, por sua vez, varia ao longo do tempo. Para o último, a endogeneidade da oferta de moeda se apresenta não por meio de alterações na taxa de juros, mas sim por meio da adequação da oferta de moeda a sua demanda representada pela influência conjunta do produto nominal com a preferência pela liquidez dos agentes. Assim sendo, diferentemente dos primeiros modelos, neste último a exogeneidade da taxa de juros nominal é plena ${ }^{6}$.

De uma forma geral, quando se endogeniza o mark-up bancário, a capacidade de expansão do crédito por parte dos bancos não sofre nenhuma restrição quantitativa proveniente de escassez de reservas. Isto porque se considera que a política monetária é realizada somente por meio da definição da taxa básica de juros. Com isso, a definição do mark-up bancário sofre influência da capacidade de autofinanciamento da firma, da preferência pela liquidez dos bancos

(6) Existe uma rica discussão teórica dentro da escola Pós-keynesiana com relação às condições e ao grau pelo qual a endogenia da moeda se manifesta nas economias capitalistas modernas. De um lado, os teóricos "Horizontalistas" que percebem a moeda como sendo plenamente endógena - Hipótese Kaldor-Moore - devido à necessidade da Autoridade Monetária de suprir a demanda por moeda dos Bancos Comerciais para evitar, desse modo, a ocorrência de crises sistêmicas. Por outro, os teóricos "Estruturalistas", que vêem a endogenia da moeda como sendo parcial, visto que a Autoridade Monetária não necessariamente acomoda toda a demanda por moeda requerida pelos Bancos Comerciais, uma vez que a possibilidade de crise sistêmica, embora presente, é vista como um evento raro na história do capitalismo. Para maiores detalhes sobre esse rico tema ver, por exemplo, Kaldor (1982, 1985), Moore (1983, 1985, 1988), Freitas (1999), Fiocca (2000) e Paula (2003). 
e do encarecimento da taxa de captação de recursos, dada pela taxa de juros básica controlada pela autoridade monetária. ${ }^{7}$

Tendo isso em mente, o presente trabalho tem como objetivo dar uma contribuição à teoria Pós-keynesiana do crescimento e da distribuição funcional da renda através do desenvolvimento de um modelo no qual a moeda, via endogenização do mark-up bancário, interage com o processo de inovação tecnológica. Criando, com isso, uma série de efeitos retroalimentadores nas relações de causalidades existentes entre o lado monetário e o lado real da economia. Alterando, desta forma, a dinâmica das principais variáveis macroeconômicas.

Ademais, cabe enfatizar que, diferentemente de seus similares, a incorporação da endogeneidade da oferta monetária no presente modelo se dá pela influência do sistema financeiro no processo de acumulação de capital via um mecanismo peculiar de inspiração schumpeteriana. $O$ processo de inovação tecnológica cria uma maior demanda por crédito que, por sua vez, permite aos bancos oligopolistas aumentarem os seus mark-ups sobre a taxa básica de juros que varia em decorrência da política monetária -, influenciando dessa forma a dinâmica de distribuição de renda e acumulação de capital da economia.

Dito isso, o artigo está organizado do seguinte modo: na seção 1, a estrutura do modelo é especificada; na seção 2, o comportamento do modelo no curto prazo é estabelecido enquanto, na seção 3, analisa-se o seu comportamento no longo prazo com base em duas variáveis de estado - a parcela salarial e o estoque de capital em unidades de oferta de trabalho eficiente. A seção 4 apresenta uma análise qualitativa de equilíbrios múltiplos, enfatizando uma onde prevalece um equilíbrio instável do tipo ponto de sela conjuntamente com um equilíbrio baseado em espirais amortecidas. Por fim, as principais conclusões geradas pelo modelo.

\section{Estrutura do Modelo ${ }^{8}$}

Consideremos uma economia fechada, onde firmas oligopolistas financiadas por bancos também oligopolistas produzem um único bem utilizável tanto para consumo quanto para investimentos. Para produzir esse bem, utilizamse somente dois fatores homogêneos de produção, capital e trabalho. Sendo a tecnologia de produção de coeficientes fixos, como evidenciado pela seguinte função de Leontief:

(7) Rousseas (1985) argumenta que os bancos determinam o preço dos empréstimos através da especificação de um mark-up sobre o custo de obtenção de fundos.

(8) O modelo apresentado a seguir baseia-se, em parte, em Lima (1999). 


$$
Q=\min \left[K u_{k} ; L / q\right]
$$

Onde $Q$ é o nível do produto, $K$ é o estoque de capital, ${ }^{9} u_{k}$ é a relação produto potencial-capital, $L$ é o nível de emprego e $q$ é a relação trabalhoproduto. A pressuposição de coeficientes fixos de produção, isto é, de elasticidade de substituição entre capital e trabalho igual a zero, pode ser explicada pela rigidez tecnológica que imputa a um fator de produção características únicas não presentes ao outro fator.

Assume-se que a economia opera com excesso de capacidade em termos de capital, o que as impede de alcançar sua produção potencial. Por sua vez, a inexistência de contratos de longa duração ou de custos de demissão, contratação e treinamento de mão-de-obra permite que as firmas utilizem a mão-de-obra na exata medida de suas necessidades representadas, dada a relação trabalho-produto, pela demanda por seu produto. Dessa forma, o nível de emprego pode ser apresentado pela seguinte equação:

$$
L=q Q
$$

Implicando que dada a relação trabalho-produto, para cada nível de produção existe um único e ótimo nível correspondente de emprego.

Os planos de acumulação da firma podem ser descritos pela seguinte relação funcional:

$$
\frac{I}{K}^{d}=\alpha+\beta(r-i)+\tau . \hat{\Gamma}
$$

Onde $\frac{I}{K}^{d}$ é a acumulação desejada das firmas como fração do estoque de capital, $\alpha$ é o parâmetro (positivo) autônomo de acumulação, $\beta$ e $\tau$ são, respectivamente, parâmetros positivos de sensibilidade do diferencial da taxa de lucro com a taxa de juros bancária e da taxa de inovação tecnológica poupadora de mão-de-obra.

Diferentemente de Lima (1999), que segue Rowthorn (1981) e Dutt (1984, 1990), nós não assumimos que a acumulação desejada depende positivamente da utilização da capacidade, como forma de possibilitar à firma uma margem ociosa do seu capital produtivo. Visando, desse modo, a inibir, via aumento da produção e conseqüente queda de preços, entradas de firmas concorrentes; assim como de se precaver de variações inesperadas da demanda efetiva.

(9) Por simplificação, considera-se que o capital não se deprecia com o passar do tempo. 
Mas, sim, assumimos que a acumulação desejada depende positivamente do diferencial da taxa de lucro com a taxa de juros bancária. Expressando dessa forma uma faceta do processo decisório da firma que, ceteris paribus, investirá em ativos reais se o retorno a ser obtido por estes, dado pela taxa de lucro, for superior ao retorno proveniente de investimentos em ativos financeiros, dado pela taxa de juros bancária. Ou, diferentemente, se o retorno do investimento em ativos reais for superior ao custo do crédito necessário para se efetuar tal investimento.

Com efeito, diferenciamos de Bhaduri e Marglin (1990) que supõem que a acumulação desejada depende linearmente e isoladamente da taxa de lucro e de Lima (1999), que assume que ela depende não linearmente do inverso da parcela dos salários. Pois agora assumimos que a acumulação desejada depende não linearmente do diferencial entre a taxa de lucro e a taxa de juros bancária, ambas expressas em termos da parcela dos salários na renda.

Finalmente, assumimos que a acumulação desejada depende positivamente da taxa de inovação tecnológica, $\hat{\Gamma}$. Diversos argumentos podem ser arrolados para tal dependência, como o de Dutt (1994), que resgata a idéia de Kalecki (1971) de que, quanto maior a taxa de progresso tecnológico, mais rentável se torna o investimento em novos equipamentos; assim como o de Schumpeter (1934, 1942), de que o processo de inovação iniciado por uma firma acaba, com o tempo, se espraiando para as demais abrindo, com isso, novas oportunidades de investimento; e com o argumento de Nelson e Winter (1982), de que o comportamento do investimento é fortemente influenciado pela dinâmica do progresso tecnológico.

A taxa de inovação tecnológica, por sua vez, é definida como sendo influenciada positivamente pela parcela salarial por meio da ponderação do parâmetro de sensibilidade $\psi$, como mostrado abaixo:

$$
\hat{\Gamma}=\psi \cdot S
$$

Cabe ressaltar, por fim, que devido à relação produto potencial-capital permanecer constante, indiferentemente da taxa de inovação tecnológica, esta é considerada neutra no sentido de Harrod.

Na mesma linha dos trabalhos de Kalecki (1971), Kaldor (1956), Marx (1971), Pasinetti (1962) e Robinson (1956, 1962), a economia é habitada por duas classes sociais, trabalhadores e capitalistas. Os primeiros ofertam mão-de-obra em troca de salários, os quais são totalmente gastos em consumo; ${ }^{10}$ os segundos poupam uma parcela $s$ sobre a taxa de lucro, que nada mais é do que todo o excedente da renda não despendido com a mão-de-obra.

(10) Pode-se também considerar, sem nenhuma alteração nesse pressuposto, que a poupança realizada por uma parte dos trabalhadores é compensada pela despoupança da outra parte dos trabalhadores. 
Baseado nisso, o produto agregado dessa economia pode ser visto como sendo a soma da remuneração dos trabalhadores, ou seja, do produto do salário real com a quantidade de trabalho empregado e com a rentabilidade do capital utilizado na produção, entendido como o valor de retorno do estoque de capital. Sendo assim, a divisão funcional da renda é dada por:

$$
Q=(W / \mathrm{P}) L+r K
$$

Sendo: $W$ é o salário nominal, $\mathrm{P}$ é o nível de preços e $r$ é a taxa de lucros definida como o fluxo monetário de lucros dividido pelo estoque de capital valorado pelo nível de preço do produto. Usando as equações (2), (5) e (8), achase a seguinte expressão da parcela salarial:

$$
S=(W / P) \cdot q
$$

Onde $W / P$ é o salário real.

A parcela salarial assume valores entre 0 e 1 , mais precisamente $0<S<1$, de forma que a parcela dos lucros na renda, $m$, pode ser escrita da seguinte forma:

$$
m=1-S
$$

A taxa de lucro pode ser expressa em função da parcela salarial e do grau de utilização da capacidade produtiva; mais precisamente temos:

$$
r=m . u
$$

No qual $u$ é o grau de utilização da capacidade, $u=Q / K$.

A taxa de inflação, por sua vez, ocorre em decorrência de conflitos distributivos, sendo o mecanismo de ajuste entre demandas distributivas incompatíveis. Devido à existência de firmas oligopolistas, a variação dos preços se dará sempre que o mark-up desejado for maior que o mark-up efetivo. A equação (6) nos informa que dada a produtividade do trabalho ou igualmente a relação trabalho-produto, o mark-up efetivo é inversamente relacionado com a parcela salarial de maneira que o hiato existente entre o mark-up desejado e o efetivo pode ser representado pela diferença entre a parcela salarial efetiva e a parcela salarial determinada pelo mark-up desejado. Formalmente temos:

$$
\hat{\mathrm{P}}=\rho\left[S-S_{f}\right]
$$

Onde $\hat{\mathrm{P}}$ é a taxa proporcional de variação no preço, $(d P / d t)(1 / P) ; \rho$ é um parâmetro de sensibilidade maior que 0 e menor ou igual a 1 e $S_{f}$ é a parcela salarial determinada pelo mark-up desejado. Baseando-se em Kalecki (1971), o preço cobrado pela firma é determinado por meio da definição de um mark-up sobre os custos primários de produção. Matematicamente, temos: 


$$
\mathrm{P}=\left(1+\mathrm{Z}_{f}\right) W \cdot q
$$

Sendo: $\mathrm{Z}_{f}$ é o mark-up da firma.

No curto prazo, a relação trabalho-produto, $q$, é fixa. Porém, no longo prazo, ela diminuirá na medida em que aumentar a taxa de inovação tecnológica, $\hat{\Gamma}$, ou seja:

$$
\hat{q}=-\psi \cdot S
$$

Onde $\hat{q}$ é a taxa de variação proporcional da relação trabalho-produto.

A parcela salarial determinada pelo mark-up desejado pela firma, $S_{f}$, depende inversamente do grau de aquecimento no mercado de bens, sendo expressa pela seguinte equação:

$$
S_{f}=\theta-\varphi u
$$

Nos quais $\theta$ e $\varphi$ são parâmetros positivos. De acordo com Eichner (1976), firmas podem desejar em momentos de expansão gerar recursos próprios para financiar seus investimentos que, como bem lembram Gordon et alii (1984), será tanto maior quanto menor for a elasticidade percebida da demanda, a qual depende negativamente da concentração de mercado e dos competidores potenciais percebidos como restringidos pelas suas capacidades produtivas.

Por sua vez, a taxa proporcional de variação do salário nominal cresce com o passar do tempo de acordo com o hiato entre a parcela salarial desejada pelos trabalhadores, $S_{W}$ e a parcela efetiva, $S$; ponderados positivamente pelo parâmetro de sensibilidade $\lambda$, como demonstrado pela seguinte equação:

$$
\hat{W}=\lambda\left[S_{W}-S\right]
$$

A parcela salarial desejada pelos trabalhadores será tanto maior quanto maior for o poder de barganha dos trabalhadores vis-à-vis ao dos capitalistas. Esse poder de barganha, por seu lado, aumentará sempre que a taxa de emprego aumentar. Formalmente temos:

$$
S_{W}=\varepsilon E
$$

No qual $\varepsilon$ é um parâmetro de sensibilidade positivo e $E$ é a taxa de emprego entendida como a razão entre o nível de emprego e a oferta de emprego $(L / N)$. A taxa de emprego está relacionada ao mercado de bens segundo a seguinte relação funcional:

$$
E=u . k
$$


Sendo que $k$ é a razão entre o estoque de capital e a oferta de trabalho em unidades de produtividade, como demonstrado na equação (16) abaixo.

$$
k=K /(N / q)
$$

A taxa de crescimento proporcional da oferta de trabalho é aqui modelada como aumentando a uma taxa exógena $\eta$, ou seja:

$$
\hat{N}=\eta
$$

A taxa básica de juros, $i_{\mathrm{B}}$, é utilizada pela autoridade monetária como instrumento de controle inflacionário. Sempre que a taxa de inflação aumentar, a autoridade monetária elevará pari passu a taxa básica de juros, como forma de conter o aumento da inflação ${ }^{11}$. Formalmente temos:

$$
i_{\mathrm{B}}=\phi \cdot \hat{\mathrm{P}}
$$

Onde: $\phi$ é um parâmetro positivo de sensibilidade da taxa básica de juros com relação à taxa de inflação.

A taxa de juros $i$ dessa economia é determinada por meio de um mark-up bancário $Z_{B}$ sobre a taxa de juros básica administrada pelo Banco Central, $i_{\mathrm{B}}$. Como pode ser evidenciado pela seguinte equação:

$$
i=\mathrm{Z}_{B} i_{\mathrm{B}}
$$

Inspirando-se em Schumpeter (1934), cujo crédito bancário depende da necessidade dos empresários inovadores em obter adiantamentos para financiar suas inovações, o mark-up bancário é aqui modelado como estando em função da taxa de inovação tecnológica. Sendo assim, quanto maior for a taxa de inovação tecnológica maior será a demanda por crédito e em melhores condições os bancos se encontrarão para aumentar suas margens de mark-up. Essa relação é formalmente denotada como segue:

$$
\mathrm{Z}_{B}=\mu . \hat{\Gamma}
$$

Sendo $\mu$ um parâmetro positivo de sensibilidade do mark-up bancário com relação à demanda por crédito, vale dizer, com relação à taxa de progresso tecnológico.

Como as firmas operam com excesso de capacidade em termos de capital, elas ajustam os seus graus de utilização da capacidade de forma a produzir na

(11) Estamos considerando no presente modelo que o governo utiliza como único instrumento de combate ao processo inflacionário a taxa básica de juros, abstendo-se de utilizar qualquer tipo de controle sobre a quantidade de moeda, assim como do uso da política fiscal.

Economia e Sociedade, Campinas, v. 17, n. 1 (32), p. 29-51, abr. 2008. 
exata medida para satisfazer a demanda efetiva. Equalizando dessa forma, o investimento desejado e a poupança segundo a seguinte equação:

$$
\frac{I}{K}=s r
$$

Na qual $\frac{I}{K}$ é a taxa de crescimento proporcional do estoque de capital e $s$ a propensão marginal a poupar dos capitalistas.

\section{Comportamento do Modelo no curto prazo}

No curto prazo assumimos que a variação do estoque de capital, $K$; da oferta de trabalho, $N$; da relação trabalho-produto, $q$; do nível de preço, $\mathrm{P}$ e do salário nominal, $W$, são todas iguais a zero.

No equilíbrio, variações no grau de utilização da capacidade produtiva farão com que a taxa de crescimento proporcional do estoque de capital seja igual à taxa proporcional de acumulação desejada das firmas, ou seja: $I / K=I^{d} / K$.

Assim temos que, no equilíbrio: $s r=\alpha+\beta(r-i)+\psi \cdot \hat{\Gamma}$. Usando as equações (3), (4), (7), (8), (9), (12), (18), (19), (20) e (21) pode-se determinar o valor de equilíbrio de curto prazo do grau de utilização da capacidade, $u^{*}$, como segue:

$$
u^{*}=\frac{-A \cdot S^{2}+B . S+\alpha}{(s-\beta)(1-S)+C \cdot S}
$$

Sendo: $\quad A \equiv \beta \mu \phi \psi \rho ; \quad B \equiv \beta \mu \phi \psi \rho \theta+\tau \psi \quad$ e $\quad C \equiv \beta \mu \phi \psi \rho \varphi$.

Assumindo a hipótese usual de que a propensão marginal a poupar é maior que a propensão marginal a investir ${ }^{12}$ e dado que $C>0$, garantimos que o denominador dessa expressão seja positivo. Como os parâmetros constituidores de $A$ e $B$ são todos positivos, temos que $A>0, B>0$ e o grau de utilização da capacidade produtiva de equilíbrio é uma parábola com a concavidade voltada para baixo e intercepto positivo igual a $\alpha$. A variação de $u^{*}$ com relação às variações na parcela salarial, $\partial u^{*} / \partial S$, é dada por:

$$
\frac{\partial u^{*}}{\partial S}=\frac{D \cdot S^{2}-F \cdot S+G}{[(s-\beta)(1-S)+C \cdot S]^{2}}
$$

(12) Essa hipótese é necessária para garantir a estabilidade da posição de equilíbrio de curto prazo dos modelos keynesianos, pois, na ausência dela, desequilíbrios no mercado de bens tenderão a ser amplificados com o passar do tempo (cf. Bhaduri; Marglin, 1990). 
Onde:

$$
D \equiv A .(s-\beta)-A . C ; F \equiv 2 A(s-\beta) ; G \equiv(B+\alpha)(s-\beta)-\alpha . C
$$

Como pode ser visto, o denominador dessa expressão é sempre positivo para qualquer valor da parcela salarial, por sua vez, o numerador apresenta valores ambíguos tanto para $D$ quanto para $G$, sendo que para $F$ é positivo, $F>0$, uma vez que supusemos que a propensão marginal a poupar é maior que a investir. Assumimos aqui que os parâmetros $D$ e $G$ assumem valores positivos, ou seja, $D>0$ e $G>0$. A suposição de $D>0$, implica a necessidade de assumirmos que o setor financeiro produz uma influência menor sobre a decisão de investir dos capitalistas do que a diferença entre as propensões marginais a poupar e a investir. Feitas essas considerações, a variação de $u^{*}$ com relação a $S$ é uma parábola com a concavidade voltada para cima, com o intercepto $G$ positivo e, depois de algumas convenientes restrições nos parâmetros, com o ponto de mínimo em $S^{*}$.

A taxa de juros bancária sofre uma influência conjunta da taxa do progresso tecnológico e da taxa básica de juros. Esta última, por sua vez, é influenciada pela taxa de inflação e pelo grau de utilização da capacidade de equilíbrio. Em vista disso, a taxa de juros cobrada pelos bancos se comporta de acordo com a seguinte expressão:

$$
i=(\mu \phi \psi \rho) \cdot S^{2}-(\mu \phi \psi \rho \theta) \cdot S+(\mu \phi \psi \rho \varphi) u . S
$$

Ao substituirmos, nessa equação, o grau de utilização da capacidade de equilíbrio, equação (22), pode-se perceber o comportamento da taxa de juros bancária de equilíbrio, isto é, da taxa de juros compatível com o equilíbrio entre a taxa desejada de acumulação das firmas e a taxa de crescimento do estoque de capital. Tal comportamento se dá de acordo com a seguinte equação:

$$
i^{*}=\frac{\Psi . S^{3}+\chi \cdot S^{2}+\pi . S}{(s-\beta)(1-S)+C . S}
$$

Onde: $\Psi \equiv C(\mu \phi \psi \rho)-(\mu \phi \psi \rho)(s-\beta)-A(\mu \phi \psi \rho \varphi)$

$$
\begin{aligned}
& \chi \equiv(\mu \phi \psi \rho \theta)(s-\beta)+B(\mu \phi \psi \rho \varphi)-C(\mu \phi \psi \rho \theta) \\
& \pi \equiv(\alpha \mu \phi \psi \rho \varphi)-(\mu \phi \psi \rho \theta)(s-\beta)
\end{aligned}
$$

Como pode ser observado, a taxa de juros de equilíbrio varia cubicamente em relação a alterações na parcela salarial. Se supusermos que os parâmetros assumem valores de tal forma a fazer com que: $\Psi<0, \chi>0, \pi>0$ e $S^{*}$ corresponda ao ponto de inflexão da função $i^{*}$, então, a taxa de juros bancária de equilíbrio aumentará a taxas crescentes e depois a taxas decrescentes até atingir o seu ponto de máximo correspondente a $S=1$. 
A taxa de crescimento da economia no equilíbrio, $I^{*} / K$, pode ser encontrada utilizando-se as equações (7), (8), (21) e (22):

$$
\frac{I}{K}^{*}=\frac{s A S^{3}-H S^{2}+M S+s \alpha}{(s-\beta)(1-S)+C S}
$$

Sendo: $H \equiv s(A+B)$ e $M \equiv s(B-\alpha)$

Uma vez que $A>0$ e admitindo que $0<\alpha<B$, então os parâmetros $H$ e $M$ são positivos, ou seja, $H>0$ e $M>0$. Nessas condições, a equação varia cubicamente, inicialmente a taxas decrescentes e posteriormente a taxas crescentes. Ademais, ao assumir algumas convenientes restrições nos pesos dos parâmetros, é possível ajustar o comportamento da função de forma a que o seu ponto de inflexão corresponda ao nível $S^{*}$ da parcela salarial e o seu ponto de máximo, ao nível $S=1$.

Derivando a equação (26) com relação a $S$, é possível evidenciar o comportamento da taxa de acumulação com relação a variações na parcela salarial, como segue:

$$
\frac{\partial\left(I^{*} / K\right)}{\partial S}=\frac{R S^{3}+T S^{2}+V S+X}{[(s-\beta)(1-S)+C S]^{2}}
$$

Onde: $\partial\left(I^{*} / K\right) / \partial S$ é a variação da taxa proporcional de acumulação em relação a mudanças na parcela salarial e $R \equiv 2 s A[C-(s-\beta)]$; $T \equiv(3 s A+H)(s-\beta)-H C ; \quad V \equiv M[(s-\beta)-C] \quad$ e $X \equiv s \alpha C-[(s-\beta)(2 H+s \alpha)]$.

A equação acima deduzida demonstra que a taxa proporcional de acumulação varia não linearmente com relação a mudanças na parcela salarial. Mais precisamente, e em razão das considerações feitas anteriormente, temos que: $R<0, T>0, V>0$ e $X<0$. Supondo que os parâmetros assumem valores de forma que $S^{*}$ corresponda ao ponto de máximo da função, a taxa de acumulação variará cubicamente de maneira que, partindo do intercepto negativo $X$, crescerá inicialmente a taxas crescentes e, posteriormente, a taxas decrescentes até atingir o seu ponto de máximo, equivalente a $S^{*}$, decrescendo então monotonicamente a partir desse ponto.

Sob estas restrições paramétricas, é possível dividir o domínio distributivo em duas regiões denominadas como PL e WL em alusão, respectivamente, aos regimes de Profit-Led e Wage-Led. Na região PL, compreendida no intervalo $0<S<S^{*}$, distribuições de renda em prol dos trabalhadores diminuem a parcela 
dos lucros na renda que, no entanto, ainda se encontra a níveis consideravelmente altos. Esse fato, corroborado pelo aumento do grau de utilização e, consequientemente da taxa de lucro, mais do que compensa o aumento da taxa de juros bancária, de forma que a taxa de acumulação do capital é positiva nessa região. Por outro lado, na região WL, compreendida no intervalo entre $S^{*}<S<1$, aumentos distributivos em prol dos trabalhadores exercem uma diminuição no grau de utilização da capacidade produtiva, da parcela dos lucros na renda, da taxa de lucro e, como a taxa de juros bancária continua aumentando, a taxa de acumulação do capital decresce nessa região.

\section{Comportamento do Modelo no longo prazo}

No longo prazo, a economia se move devido a variações no estoque de capital, na oferta de trabalho, na relação trabalho-produto, no nível de preço e no salário nominal, estando sempre o grau de utilização da capacidade, a taxa de acumulação e a taxa de juros bancária em equilíbrio.

A partir das seguintes definições da parcela salarial, $S \equiv(W / P) q$ e da razão entre o estoque de capital e a oferta de trabalho em unidades de produtividade, $k \equiv K /(N / q)$; pode-se acompanhar o comportamento dinâmico do sistema.

Linearizando ambas as equações e derivando-as em relação ao tempo, chega-se ao seguinte sistema bidimensional de equações diferenciais não-lineares:

$$
\begin{array}{r}
\hat{S}=\hat{W}-\hat{\mathrm{P}}+\hat{q} \\
\hat{k}=\hat{K}+\hat{q}-\hat{N}
\end{array}
$$

Onde: $\hat{S}=\partial S / S ; \hat{k}=\partial k / K$ e assim por diante.

Substituindo as equações (9), (11), (12), (13), (14) e (15) na equação (28), podemos apresentar a taxa de variação proporcional da parcela salarial, em função do estoque de capital em unidades de oferta de trabalho eficiente, $k$ e do grau de utilização da capacidade produtiva de equilíbrio, $u^{*}$; que, por sua vez, encontra-se unicamente em função da parcela salarial, $S$. Sendo assim, temos que:

$$
\hat{S}=u^{*}[(\lambda \varepsilon) k-\rho \varphi]-(\lambda+\psi+\rho) S+\rho \theta
$$

Igualando a taxa de crescimento proporcional da parcela salarial a zero e aplicando a derivada total ao resultante dessa expressão, é possível determinar a inclinação do lócus $\hat{S}=0$ através da seguinte equação:

$$
\frac{\partial k}{\partial S}=\frac{[\rho \varphi-(\lambda \varepsilon) \cdot k] \cdot\left(\partial u^{*} / \partial S\right)+(\lambda+\psi+\rho)}{u^{*} \lambda \varepsilon}
$$


O denominador dessa expressão é sempre positivo para qualquer valor da parcela salarial, isto é, $u^{*}>0, \forall 0<S<1$. A taxa de variação do grau de utilização de equilíbrio com relação à parcela salarial, $\partial u^{*} / \partial S$, é uma parábola com a concavidade voltada para cima. Considerando a hipótese bastante razoável de que os preços se ajustam mais rapidamente do que os salários nominais tem-se que: $[\rho \varphi-(\lambda \varepsilon) \cdot k]>0$. Se considerarmos que $[\rho \varphi-(\lambda \varepsilon) \cdot k] \cdot\left(\partial u^{*} / \partial S\right)>\lambda+\psi+\rho$; então a inclinação do lócus $\hat{S}=0$ será negativa, $\partial k / \partial S<0$, para valores baixos da parcela salarial $\left(0<S<S^{*}\right)$ e positiva, $\partial k / \partial S>0$, para valores altos da parcela salarial $\left(S^{*}<S<1\right)$. Ademais, como $\partial u^{*} / \partial S$ é uma função quadrática na parcela salarial, existem até duas raízes reais que satisfazem a equação quando se considera o lócus $\hat{S}=0$.

Sabe-se que: $I \equiv \frac{\partial K}{\partial t} \Rightarrow \frac{I}{K} \equiv \hat{K} \equiv s r=s(1-S) u$. Ao substituir essa equação juntamente com as equações (11) e (17) na equação (29), pode-se apresentar a taxa de variação proporcional do estoque de capital em unidades de oferta de trabalho eficiente, $\hat{k}$, em função de $S$, como demonstra a equação abaixo:

$$
\hat{k}=\frac{s A \cdot S^{3}+\Omega \cdot S^{2}+\Sigma . S+\Lambda}{(s-\beta)(1-S)+C . S}
$$

Sendo: $\Omega \equiv C \psi-H-\psi(s-\beta) ; \quad \Sigma \equiv J+\eta C+\psi(s-\beta)-\eta(s-\beta)$; $\Lambda \equiv s \alpha+\eta(s-\beta)$ e $\eta$ é a taxa positiva de crescimento exógena da força de trabalho.

Assumindo que os parâmetros adquirem valores de forma que: $\Omega<0$, $\Sigma>0$ e $\Lambda>0$ e como a equação (32) é cúbica, existem até três raízes reais que satisfazem a igualdade quando é definido o lócus $\hat{k}=0$. No entanto, apenas duas dessas raízes assumem valores positivos no espaço $(k-S)$. Aplicando a derivada total ao lócus $\hat{k}=0$, verifica-se também que $k$ é insensível a variações em $S$, podendo então o lócus $\hat{k}=0$ ser representado por meio de duas retas verticais em relação ao eixo das ordenadas.

Diante disto, temos um sistema bidimensional de equações diferenciais não-lineares $\hat{S}$ e $\hat{k}$ que descrevem, respectivamente, o comportamento da parcela salarial, $S$, e do estoque de capital em unidades de oferta eficiente de trabalho, $k$, ao longo do tempo. 
Derivando, por fim, as equações diferenciais (28) e (29) com relação a $S$ e $k$ e efetuando as substituições necessárias, obtemos a seguinte matriz Jacobiana $|J|$ de derivadas parciais:

$$
\begin{gathered}
J_{11} \equiv \partial \hat{S} / \partial S=\left(\partial u^{*} / \partial S\right)(\lambda \varepsilon \cdot k-\rho \varphi)-(\lambda+\psi+\rho) \\
J_{12} \equiv \partial \hat{S} / \partial k=\lambda \varepsilon \cdot u^{*}>0 \\
J_{21} \equiv \partial \hat{k} / \partial S=\left[\partial\left(I^{*} / K\right) / \partial S\right]+\psi \\
J_{22} \equiv \partial \hat{k} / \partial k=0
\end{gathered}
$$

A expressão (33) nos mostra que a variação da taxa de crescimento proporcional da parcela salarial com relação a ela mesma, $\partial \hat{S} / \partial S$, pode ser positiva ou negativa, dependendo para sua definição de determinar o impacto da parcela salarial sobre o grau de utilização da capacidade no equilíbrio, $\partial u^{*} / \partial S$. A variação da taxa proporcional da parcela salarial em decorrência de mudanças no estoque de capital em unidades de eficiência de trabalho, equação (34), assume valores positivos em todo o espaço relevante $(k-S)$. Isto porque o aumento de $k$ eleva numa intensidade ditada por $\lambda$ e $\varepsilon$ a taxa de emprego e o aumento da parcela salarial requerida pelos trabalhadores, levando, por consequiência, ao aumento da taxa de crescimento dos salários nominais. Esse efeito ocorre ao mesmo tempo em que se eleva a parcela salarial implicada pelo mark-up desejado, provocando com isso a queda na taxa de variação dos preços; efeitos esses que, em seu conjunto, levam ao aumento dos salários reais.

A existência de ambigüidade também aparece na equação (35). Devido às restrições paramétricas assumidas anteriormente, o sinal de $\partial \hat{k} / \partial S$ será positivo se a parcela distributiva for baixa, ou seja, se estiver compreendida entre $0<S<S^{*}$; e negativo, se estiver compreendida no intervalo entre $S^{*}<S<1$. Por fim, na equação (36), a variação de $k$ não impacta a parcela salarial, $S$ e nem o grau de utilização da capacidade, $u^{*}$; não influenciando, portanto, a taxa proporcional do estoque de capital em unidades de oferta de trabalho eficiente, $\hat{k}$.

Podemos agora analisar as propriedades de estabilidade do modelo, por meio de diagramas de fases. Lembrando que o domínio distributivo compreendido no espaço $(k-S)$ foi dividido em duas regiões - PL e WL -, através de uma bissetriz que corta a isolinha $\hat{S}=0$, no seu ponto de mínimo correspondente ao nível da parcela salarial, $S^{*}$. 


\section{Análise de equilíbrios múltiplos}

Uma análise da estabilidade local do sistema pode ser efetuada calculando o traço, $\operatorname{Tr}|J|$, e o determinante, $\operatorname{Det}|J|$, da matriz Jacobiana $|J|$. Assim, o sistema será instável se $\operatorname{Tr}|J|>0$ e o $\operatorname{Det}|J|>0$; instável do tipo ponto de sela se, $\operatorname{Tr}|J|>0$ e o $\operatorname{Det}|J|<0$; ou estável se o $\operatorname{Tr}|J|<0$ e o $\operatorname{Det}|J|>0$ (cf. Takayama, 1993 e De La Fuente, 2000, cap. 10).

Em razão da existência de não-linearidades no modelo e do fato de a análise que se pretende fazer ser qualitativa, existem diversas configurações possíveis nas quais se podem obter equilíbrios múltiplos no espaço $(k-S)$. Sendo assim, escolhemos uma na qual há um equilíbrio instável do tipo ponto de sela (saddle path) na região PL e um equilíbrio estável baseado em uma espiral amortecida (stable spiral path) na região WL.

Na região $\mathrm{PL}$, compreendida no intervalo $0<S<S^{*}$, prevalece um regime de profit-led. Distribuições de renda em prol dos trabalhadores diminuem a parcela dos lucros na renda e aumentam o grau de utilização da capacidade, ao mesmo tempo em que elevam o custo da mão-de-obra e, por consequiência, a taxa de inovação tecnológica. Uma vez que a parcela dos lucros na renda, apesar de diminuindo, continua elevada e, como o grau de utilização da capacidade está se elevando, a taxa de lucro é positiva nessa região, embora decrescente.

No sistema financeiro, o aumento da parcela salarial gera um duplo impacto sobre a taxa de juros bancária. Por um lado, esse aumento eleva a taxa de progresso tecnológico, aumentando com isso o mark-up bancário como consequiência da elevação da demanda por crédito por parte das firmas inovadoras. Por outro lado, o aumento da parcela salarial amplia o grau de utilização da capacidade, elevando assim o nível de preços e, como reação a esse aumento, a taxa de juros básica controlada pela autoridade monetária. Esses dois efeitos, por fim, levam a um aumento da taxa de juros cobrada pelos bancos.

Ademais, como a taxa de progresso tecnológico, a propensão autônoma a investir e o diferencial da taxa de lucro com relação à taxa de juros são positivos, a taxa de acumulação aumenta em toda a região PL, embora inicialmente a taxas crescentes e, posteriormente, a taxas decrescentes. Esses efeitos em seu conjunto levam, por fim, a uma diminuição do estoque de capital em unidades de eficiência de trabalho, $k$, visto que o impacto negativo da oferta exógena de trabalho, somada com a diminuição da relação trabalho-produto, mais do que compensa o aumento da taxa de acumulação desejada.

Na região WL, compreendida no intervalo entre $S^{*}<S<1$, prevalece um regime de wage-led. Aumentos distributivos em prol dos trabalhadores 
comprimem a parcela dos lucros na renda e diminuem o grau de utilização da capacidade, levando assim a uma queda na taxa de lucro. Outro fator a ser levado em consideração é que, ao encarecer o custo da mão-de-obra, o aumento da parcela salarial eleva a taxa de inovação tecnológica. Como há uma queda no grau de utilização da capacidade produtiva, a taxa de emprego e, conseqüentemente, a parcela salarial desejada pelos trabalhadores, diminuem ao mesmo tempo em que a parcela salarial implicada pelo mark-up efetivo aumenta.

No sistema financeiro, duas forças opostas estão em ação. De um lado a queda no grau de utilização da capacidade enfraquece a capacidade das firmas em aumentar a sua margem desejada de mark-up, o que conduz a queda no nível de preços e, em conseqüência disso, da taxa de juros básica. De outro lado, o aumento dos custos da mão-de-obra gera pressões altistas sobre a taxa de inovação tecnológica e, dessa forma, ocorre um aumento no mark-up cobrado pelos bancos. Devido às restrições paramétricas assumidas na especificação da equação (25), a resposta do mark-up bancário ao aumento da parcela salarial é maior do que a resposta da taxa básica de juros à queda do nível de preços, de forma que a taxa de juros bancária continua aumentando nessa região, embora agora a taxas decrescentes.

Além disso, uma vez que a taxa de lucro é menor que a taxa de juros cobrada pelos bancos e como assumimos que a influência do setor financeiro é maior que a influência da propensão autônoma a investir, conjuntamente com a influência do progresso tecnológico, a taxa de acumulação de equilíbrio passa a ser negativa na medida em que a parcela salarial assume valores maiores do que $S^{*}$. A inter-relação desses efeitos faz com que a taxa da variação do grau de utilização da capacidade produtiva de equilíbrio em relação a aumentos da parcela salarial seja positiva, assim como a relação capital-oferta de trabalho em unidades de eficiência.

Em virtude de os preços se ajustarem mais rapidamente do que os salários nominais e lembrando que na região PL a $\partial u^{*} / \partial S<0, J_{12}>0$ e $J_{22}=0$, a natureza do equilíbrio dessa região dependerá da determinação do sinal de $J_{11}$. A análise dessa região nos informa que caso $\left(\partial u^{*} / \partial S\right) \cdot[(\lambda \varepsilon) k-\rho \varphi]>(\lambda+\psi+\rho)$, o traço da matriz jacobiana será positivo, $\operatorname{Tr}|J|>0$. Como nessa região $\partial\left(I^{*} / K\right) / \partial S>0$, o que faz com que $J_{21}>0$, para todo valor da parcela salarial compreendido entre 0 e 1 , então o $\operatorname{Det}|J|<0$, implicando que o sistema terá um equilíbrio instável do tipo ponto de sela. Contudo, caso $\left(\partial u^{*} / \partial S\right) \cdot[(\lambda \varepsilon) k-\rho \varphi]<(\lambda+\psi+\rho)$, o traço da matriz jacobiana será negativo, $\operatorname{Tr}|J|<0$ e como o determinante da matriz jacobiana continua sendo negativo, Det $|J|<0$, o sistema não terá equilíbrio. 
Analisando a região WL vemos que, em virtude de nessa região $\partial u^{*} / \partial S>0$, a variação proporcional da parcela salarial com relação a ela mesma será menor que zero, $J_{11}<0$ e como $J_{22}=0$, então o traço da matriz jacobiana será menor que zero, $\operatorname{Tr}|J|<0$. Uma vez que $J_{12}>0, \forall 0<S<1$, a determinação da natureza do equilíbrio nessa região dependerá do sinal de $J_{21}$. Devido ao fato de termos assumido que $\partial\left(I^{*} / K\right) / \partial S<0$ para valores da parcela salarial compreendidos nesse intervalo da parcela salarial, então se $\partial\left(I^{*} / K\right) / \partial S>\psi, J_{21}<0$ e o determinante da matriz jacobiana será maior que zero, $\operatorname{Det}|J|>0$ e o sistema terá um equilíbrio estável. Todavia, se $\partial\left(I^{*} / K\right) / \partial S<\psi, J_{21}>0$, o que implica um determinante menor que zero, $\operatorname{Det}|J|<0$ e o sistema não terá equilíbrio.

Dessa forma, existem diversas combinações possíveis, no espaço $(k-S)$, que geram algum tipo de equilíbrio. Dentre essas combinações, a Figura 1 nos mostra uma combinação onde, na região PL, há um equilíbrio instável do tipo ponto de sela e, na região WL, um equilíbrio estável baseado em uma espiral amortecida. Como ao longo de todo o domínio distributivo $J_{12}>0$ e $J_{22}=0$,

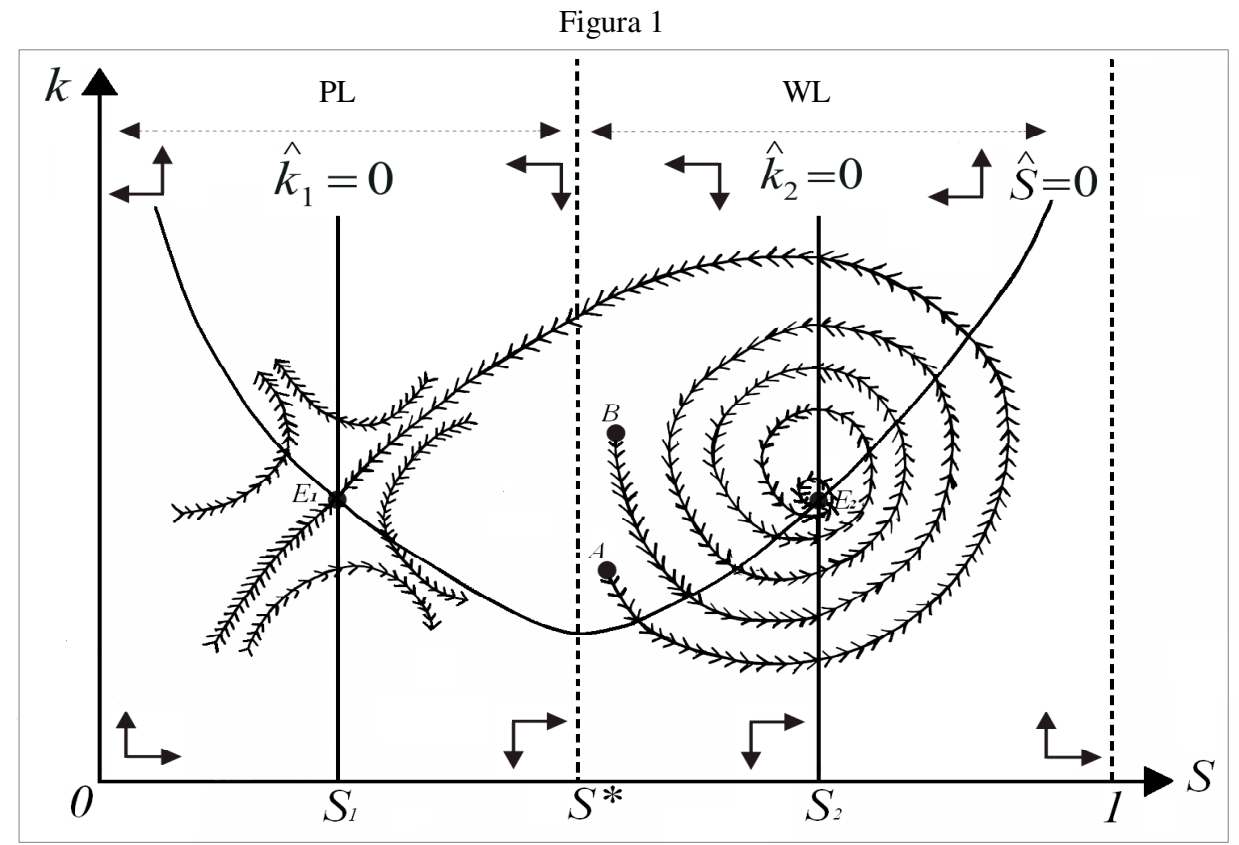

a determinação dessa configuração dependerá dos sinais de $J_{11}$ e $J_{21}$ que, por sua vez, dependem do poder de barganha existente entre capitalistas e trabalhadores; 
da sensibilidade dos preços e dos salários nominais; do peso entre a diferença da propensão marginal a poupar e a investir com relação às variáveis financeiras; das intensidades dos efeitos da inovação tecnológica e da taxa básica de juros, esta última influenciada pela variação dos preços sobre a taxa de juros bancária e, por fim, da maior influência conjunta das taxas de lucro, de juros e de progresso tecnológico sobre a propensão autônoma a investir dos capitalistas.

Sendo assim, supomos que na região PL, $J_{11}>0$, pois consideramos que a sensibilidade dos preços seja maior do que a dos salários nominais e que a influência do produto entre o grau de utilização da capacidade e os parâmetros definidores do salário real seja maior do que a soma dos parâmetros da taxa de inovação, do nível de preços e do salário nominal. Ademais, assumimos que $J_{21}>0$, isto é, que a diferença entre a propensão marginal a poupar e a investir seja maior do que o produto de todos os parâmetros definidores da taxa de juros bancária.

Para a região WL estabelecemos que $J_{11}<0$ e $J_{21}<0$. O sinal negativo do primeiro elemento da matriz jacobiana, $J_{11}$, é facilmente observável, bastando para isso recordarmos que nessa região $\partial u^{*} / \partial S>0$. O sinal negativo do segundo elemento requer que estabeleçamos, em primeiro lugar, que a diferença entre a propensão marginal a poupar e a investir seja maior do que o produto do conjunto dos parâmetros definidores da taxa de juros bancária. Em segundo lugar, é preciso assumir que o efeito da taxa de acumulação a variações na parcela salarial seja maior do que a sensibilidade da taxa de inovação tecnológica a mudanças na parcela salarial.

Analisando conjuntamente o comportamento dinâmico em todo o espaço $(k-S)$, vemos que existe um subconjunto na região WL, que caso a dinâmica econômica passe por ele, as variáveis de estado, vale dizer, a parcela salarial e o estoque de capital por unidade de oferta de trabalho eficiente, apresentarão flutuações amortecidas até atingirem o ponto de equilíbrio $\mathrm{E}_{2}$, como pode ser observado pela trajetória que sai do ponto B .

A essa zona cuja dinâmica caracterizada por flutuações amortecidas, chamaremos de zona de estabilidade e ao seu complemento de zona de instabilidade. Assim sendo, caso a dinâmica da economia se dê a partir da zona de instabilidade, o comportamento dessa economia será explosivo, a não ser que ocorra um caso muito singular no qual digamos, a partir do ponto A, a economia assuma uma dinâmica correspondente ao ponto de equilíbrio $\mathrm{E}_{1}$, na região PL, caracterizado por ser um ponto de sela de natureza eminentemente instável. 
Cabe, por fim, ressaltar que é justamente essa trajetória de sela que divide a zona de estabilidade da zona de instabilidade. Com efeito, qualquer trajetória que inicie abaixo da trajetória representada pelo ponto de sela apresentará uma dinâmica estável baseada em flutuações amortecidas e, acima dessa trajetória, apresentará uma dinâmica explosiva.

\section{Conclusões}

A introdução do papel dos bancos no financiamento das inovações tecnológicas por uma ótica schumpeteriana, isto é, através da elevação do custo do crédito bancário em decorrência do aumento da procura por adiantamentos para investimentos em inovações, traz um elemento novo na influência do progresso tecnológico sobre a dinâmica econômica.

Esta, por sua vez, se torna ainda mais complexa, quando se considera a intervenção da autoridade monetária, por meio da manipulação da taxa básica de juros, com o objetivo de manter sob controle a taxa de inflação vigente na economia. Ao se elevar o mark-up bancário e/ou a taxa básica de juros e, conseqüentemente, a taxa de juros bancária cobrada aos demandantes de crédito, surge uma série de mecanismos retroalimentadores nas relações de causalidade entre as variáveis econômicas.

Demonstramos que sob as hipóteses de que (i) a propensão marginal a poupar seja significativamente maior do que a propensão marginal a investir; (ii) a diferença entre a propensão marginal a poupar e a investir seja maior que a influência conjunta dos parâmetros financeiros; (iii) a propensão autônoma a investir e o parâmetro autônomo da parcela salarial implicada pelo mark-up efetivo sejam maiores do que o produto da diferença entre a propensão marginal a poupar e a investir, com relação ao parâmetro que mede a influência da demanda efetiva sobre a parcela salarial implicada pelo mark-up efetivo; (iv) o ajustamento do nível de preços seja mais rápido do que o ajustamento dos salários nominais; (v) o markup bancário seja mais sensível a variações na taxa de inovação tecnológica do que a taxa básica de juros o é com relação ao nível de preços, a economia aqui modelada descreve uma dinâmica complexa permeada de efeitos sobrepostos.

Com efeito, aumentos na parcela salarial elevam, num primeiro momento, o grau de utilização da capacidade, a taxa de acumulação de capital, a taxa de inovação tecnológica, a taxa de juros bancária, a taxa de emprego, o salário nominal, o nível de preço e a taxa básica de juros, ao mesmo tempo em que diminuem a taxa de variação do grau de utilização da capacidade produtiva. Num segundo momento, aumentos na parcela salarial diminuem o grau de utilização da capacidade produtiva, a taxa de variação da acumulação de capital, o nível de preço e a taxa básica da economia; enquanto ocorrem aumentos na taxa de 
variação do grau de utilização da capacidade, na taxa de acumulação de capital, na taxa de juros bancária e na taxa de inovação tecnológica.

Com relação aos regimes de acumulação, na região PL, prevalece um regime de Profit-led, isto é, um regime onde aumentos da parcela salarial diminuem a parcela dos lucros na renda, diminuindo, com isso, o estoque de capital em unidades de eficiência de trabalho. Enquanto na região WL prevalece um regime de Wage-led, isto é, um regime onde distribuições de renda prótrabalhadores apesar de comprimirem a parcela dos lucros na renda, geram aumentos no estoque de capital em unidades de eficiência de trabalho.

Além disso, a natureza não-linear desse modelo permite evidenciar a existência de flutuações amortecidas na taxa de variação do estoque de capital em unidades de eficiência de trabalho e na parcela salarial. Ademais, da mesma forma que Dutt (1994) e Lima (1999), o modelo formaliza a dinâmica de preços e salários nominais com base na existência de conflitos distributivos entre capitalistas e trabalhadores, considera a ocorrência de progresso tecnológico endógeno e confere à demanda efetiva uma influência fundamental na dinâmica econômica por intermédio da flexibilização do grau de utilização da capacidade. Mas, diferentemente destes, o sistema financeiro, por meio da ação dos bancos e da autoridade monetária, passa agora a influenciar a dinâmica econômica. Aproximando-se, dessa forma, ao tipo de economia preconizada tanto por Schumpeter quanto por Keynes.

Por fim, analisamos as condições de estabilidade do modelo, demonstrando a possibilidade de existência de equilíbrios múltiplos em torno do domínio distributivo relevante. Ilustrando o caso em que o sistema apresenta um equilíbrio instável na região onde é baixa a parcela salarial e na qual a taxa de acumulação em unidades de eficiência de trabalho é "puxada" pelo aumento dos lucros dos capitalistas; e um equilíbrio estável baseado em flutuações amortecidas na região na qual a parcela salarial é elevada e a acumulação de capital em unidades de eficiência de trabalho cresce quando a taxa de lucro dos capitalistas é comprimida.

\section{Bibliografia}

BHADURI, A.; MARGLIN, S. Unemployment and the real wage: the economic basis for contesting political ideologies. Cambridge Journal of Economics, v. 14, n. 4, 1990.

CARVALHO, F. C. Moeda, produção e acumulação: uma perspectiva pós-Keynesiana. In: MOEDAS e produção: teorias comparadas. Brasília: UnB, 1992.

DE LA FUENTE, A. Mathematical methods and models for economists. Cambridge: Cambridge University Press, 2000. 
DUTT, A. K. Stagnation, income distribution and monopoly power. Cambridge Journal of Economics, v. 8, 1984.

Accumulation, distribution and inflation in a Marxian/Post keynesian model with a rentier class. Review of Radical Political Economics, v. 21, n. 3, p. 18-26, 1989.

University Press. 1990

Growth, Distribution and uneven Development. Cambridge: Cambridge

Expectations and equilibrium: implications for Keynes, the neo-Ricardian Keynesians, and the post-Keynesians. Journal of Post Keynesian Economics, v. 14, n. 2, 1992.

On the long-run stability of capitalist economies: implications of a model of growth and distribution. In: DUTT, A. K. (Org.). New directions in analytical political economy. Aldershot: Edward Elgar, 1994.

; AMADEO, E. J. A post-Keynesian theory of growth, interest and money. In: BARANZINI, M.; HARCOURT, G. C. (Ed.). The dynamics of the wealth of nations: growth, distribution and structural change. New York: St. Martins's Press, 1993.

DOMAR, E. Capital expansion, rate of growth and employment. Econometrica, v. 14, 1946.

EICHNER, A. The megacorp and oligopoly. Cambridge: Cambridge University Press, 1976.

FIOCCA, D. A oferta de moeda na macroeconomia keynesiana. São Paulo: Paz e Terra, 2000.

FREITAS, C. P. Moeda endógena e passividade bancária: uma análise crítica da abordagem "horizontalista" e da Teoria do Circuito Monetário. Revista de Economia Política, v. 19, n. 4, p. 114-133, 1999.

GOODWIN, R. A growth cycle. In: FEINSTEIN, C. H. (Org.). Socialism, capitalism and economic growth. Cambridge: Cambridge University Press, 1967.

GORDON, D.; WEISSKOPF, T.; BOWLES, S. Long-term growth and the cyclical restoration of profitability. In: GOODWIN, R.; KRUGER, M.; VERCCELI, A. (Org.). Nonlinear models of fluctuating growth. Berlim: Spring-Verlag, 1984.

HARROD, R. An essay in dynamic theory. Economic Journal, v. 49, Mar. 1939.

KALDOR, N. Alternative theories of distribution. Review of Economic Studies, v. 23, n. 2, 1956.

A model of economic growth. Economic Journal, v. 67, 1957.

The scourge of monetarism. Oxford: Oxford University Press, 1982.

How monetarism failed. Challenge, May/Jun. p. 12-21, 1985.

KALECKI, M. The theory of economic dynamics. London: Allen \& Unwin, 1954.

Selected essays on the dynamics of the capitalist economy. Cambridge

University Press, 1971. 
KREGEL, J. A. Hamlet without the prince: Cambridge macroeconomics without money. American Economic Review, 1985.

LIMA, G. T. Progresso tecnológico endógeno, crescimento econômico e distribuição de renda. In: LIMA, G. T.; SICSÚ, J.; DE PAULA, L. F. (Org.). Macroeconomia moderna: Keynes e a economia contemporânea. Rio de Janeiro: Campus, 1999.

; MEIRELLES, A. J. Mark-up bancário, conflito distributivo e utilização da capacidade produtiva: uma macrodinâmica pós-Keynesiana. Revista Brasileira de Economia, Jan./Mar. 2003.

MARX, K. O capital. Rio de Janeiro: Civilização Brasileira, 1971.

MOORE, B. Unpacking the post Keynesian black box: banking lending and the money supply. Journal of Post Keynesian Economics, Summer 1983.

Wages, bank lending and endogeneity of credit money. In: JARSULIC, Marc (Org.). Money and macro policy. Hingham: Kluwer Academic Publisher, 1985. p. 1-28.

Horizontalists and verticalists: the macroeconomics of credit money. Cambridge: Cambridge University Press, 1988.

NELSON, R.; WINTER, S. An evolutionary theory of economic change. Cambridge: Harvard University Press, 1982.

PASINETTI, L. The rate of profit and income distribution in relation to the rate of economic growth. Review of Economic Studies, v. 29, 1962.

PAULA, L. F. Teoria horizontalista da moeda e do crédito: Crítica da crítica. Estudos Econômicos, São Paulo, v. 33, 2003.

ROBINSON, J. The accumulation of capital. London: MacMillan, 1956.

Essays in the theory of economic growth. London: MacMillan, 1962.

ROUSSEAS, S. A mark-up theory of Bank Loan Rates. Journal of Post Keynesian Economics, v. 8, n. 1, 1985.

ROWTHORN, R. Demand, real wages and economic growth. Thames Papers in Political Economy, Autumn 1981.

SCHUMPETER, J. The theory of economic development. 2. ed. New York: Harper \& Brothers, 1934.

Capitalism, socialism and democracy. New York: Harper \& Brothers, 1942.

TAKAYAMA, A. Analytical methods in economics. Michigan: University of Michigan Press, 1993.

VERCELLI, A. Methodological foundations of macroeconomics: Keynes and Lucas. Cambridge: Cambridge University Press, 1991.

WATANABE, K-I. An endogenous growth model with endogenous money supply: integration of post-Keynesian growth models. Banca Nazionale del Lavoro. Quarterly Review, n. 200, Mar. 1997. 